\title{
¿QUÉ OCURRE CUANDO COMPARAMOS DOS UNIDADES LÉXICAS SINÓNIMAS? UN ESTUDIO PSICOLINGÜÍSTICO SOBRE LA NATURALEZA DE LA SINONIMIA*
}

\author{
WHAT HAPPENS IF TWO SYNONYMS ARE COMPARED? A \\ PSYCHOLINGUISTIC STUDY ON THE NATURE OF SYNONYMY
}

\author{
MARÍA DEL CARMEN HORNO-CHÉLIZ \\ Universidad de Zaragoza, Zaragoza, España. \\ mhorno@unizar.es
}

RAQUEL TIMOR

Universidad de Zaragoza, Zaragoza, España.

raqueltiber@hotmail.com

\author{
ANTONIO SARASA \\ Universidad Complutense de Madrid, Madrid, España. \\ asarasa@gmail.com
}

\section{RESUMEN}

El presente estudio tiene como propósito estudiar, desde un punto de vista experimental, la naturaleza de la sinonimia léxica. En concreto, se pretende contestar tres preguntas diferentes: 1) ¡cómo es la relación de sinonimia, gradual o discreta?, 2) ¡es realista la percepción consciente de los hablantes sobre el grado de sinonimia entre dos ítems? y 3) ¿cómo se procesa dicha relación en el cerebro? Para ello, siguiendo a Recarte Goldaracena y Peraita Adrados (1988), se ha preparado un experimento conductual (una tarea de decisión semántica) en la que los participantes debían decir si dos ítems eran o no sinónimos. En segundo lugar, se pasaba a esos mismos sujetos un cuestionario en el que tenían que decidir, en una escala Likert de 10 posiciones, cuán sinónimas eran las mismas parejas de

*El presente trabajo se ha realizado en el marco del grupo de investigación Psylex (grupo consolidado por la Diputación General de Aragón desde 2008) y se ha beneficiado de dos proyectos de investigación del Ministerio de Economía y Competitividad: Proyecto FFI2013-45553-C3-1-P: "MovESII-VIA (variación y adquisición)” y Proyecto FFI2015-63497-P, "La interfaz Semántica/ Pragmática y la resolución de los desajustes interpretativos" (SPIRIM). Queremos agradecer públicamente la detallada lectura del manuscrito original y las acertadas sugerencias al Dr. José Manuel Igoa y a dos anónimos revisores. Los errores que hayan podido prevalecer son de responsabilidad exclusiva de los autores. 
ítems de la tarea de decisión anterior. A partir de los resultados de estas dos pruebas y de su correlación se llega a la conclusión de que la sinonimia es una relación gradual y especular que opera, en tiempo real y de modo holístico, tanto a nivel consciente (metalingüístico: el valor ofrecido en el cuestionario) como automático (procedimental: el tiempo de reacción en la tarea de decisión semántica).

Palabras clave: Sinonimia, procesamiento, semántica léxica, relaciones léxicas, psicolinguiística.

\section{ABSTRACT}

The aim of this research is the analysis of synonymy through an experimental design. Three questions have been considered: 1) is the synonymy relationship gradual or discrete? 2) is the conscious perception of the synonymy realistic? And 3) how does the brain processes this relationship? Following a previous work by Recarte Goldaracena and Peraita Adrados (1988), a lexical decision task (where participant should decide whether two items were synonyms) has been prepared. Following this task, participants were asked to mark, in a ten-Likert scale how synonyms the same partners of words were. As the result of this research, synonymy seems to be a gradual, mirrored, holistic and real relationship, which works both in the conscious and unconscious processing of language information.

Keywords: Synonym, processing, lexical semantics, lexical relationships, psycholinguistics.

Recibido: 20.06.2016. Aceptado: 24.04.2017.

\section{INTRODUCCIÓN}

La relación de sinonimia ha sido objeto de estudio de los interesados por el lenguaje y las lenguas desde, al menos, la antigüedad clásica, aunque la tradición sinonímica moderna suele considerarse que nace en el siglo XVIII, con la publicación en 1718 de la obra de Girard (v., al respecto, García Hernández, 1997). Y en el último siglo ha sido abordada desde distintos paradigmas ${ }^{1}$. En cualquier caso, pese a haber sido objeto de análisis recurrentes, dista de ser bien comprendida, por lo que todavía resulta interesante volver a ella una vez más.

Se parte de que la sinonimia es una relación léxica (esto es, entre unidades léxicas) simétrica, a diferencia de otras relaciones, como la hiponimia, por ejemplo, que es asimétrica (Murphy, 2003). Sobre cuál es la naturaleza de dicha relación, existen discrepancias. Desde el punto de vista de la gramática estructural, se trata-

${ }^{1}$ Para aquellos lectores interesados en la Lingüística Cognitiva es interesante el trabajo de Glynn y Robinson (2014), en el que se hace una revisión a los trabajos de sinonimia en este marco teórico. 
ría de una relación sobre la naturaleza semántica de los ítems léxicos. Suele basarse esta perspectiva en un análisis semántico de descomposición en rasgos, primitivos u otro tipo de unidades. Así, la sinonimia consistiría fundamentalmente en la coincidencia parcial o total (sobre esto hablaremos más adelante) de dichas unidades básicas (Lyons, 1968). Por el contrario, el análisis funcional considera que la sinonimia es una relación que se observa en uso, en la posibilidad de compartir los contextos de aparición (Arppe y Järvikivi, 2007; Glynn y Robinson, 2014; Gries y Otani, 2010). Estamos, pues, ante una relación que se puede interpretar tanto desde un punto de vista inmanente como contextual y de uso. La metodología de estudio se verá influida por ello: si los estudios que se centran en la semejanza del significado se suelen basar en la introspección (propia del lingüista o de los hablantes por medio de cuestionarios), los contextuales están irremediablemente ligados a la metodología de corpus. En este trabajo se decidió investigar sobre la sinonimia en tanto en cuanto relación de significados, sin tener en cuenta el contexto de aparición de las palabras y se ha dejado, así, para más adelante el necesario estudio de la sinonimia como fenómeno contextual. Esto no implica una postura inicial sobre la naturaleza de dicho significado léxico (conjunto de rasgos, prototipos, etc.). De hecho, se espera que los resultados de esta investigación puedan dar luz también sobre este asunto.

\section{1. ¿Cómo es la relación de sinonimia: gradual o discreta?}

Los autores que se han interesado por esta relación de sinonimia se pueden dividir en dos tipos: los que la interpretan como una relación discreta, tal que dos palabras son sinónimas o no lo son, y los que la interpretan como una relación gradual, de tal modo que lo que se debe establecer es el grado de sinonimia que presentan dos ítems concretos.

Para los primeros, el problema fundamental ha sido establecer los límites de lo que se considera sinonimia. Tienen que salvar el escollo de que, considerada como relación de identidad, la sinonimia como tal no existiría (Milner, 1989)2. En efecto, todas las piezas léxicas difieren de un modo u otro, aunque sea en aspectos como el contexto de aplicación, sus valores connotativos o la variación diatópica. De este modo, para seguir considerando esta relación, deben renunciar a esta identidad total y limitar los aspectos que necesariamente se deben compartir para que dos elementos sean sinónimos. Como señala Fruyt (1994), la sinonimia no es una relación de identidad, sino de semejanza. En caso contrario, obviamente no abundarían los términos sinónimos, pues serían un lujo excesivo para el sistema (Gau-

${ }^{2}$ Defensores de la sinonimia absoluta, sin embargo, también los hay. Se pueden mencionar, entre otros, a Salvador (1985) o a Trujillo (1996). 
ger, 1972; Casas Gómez, 1995). Además, en este marco en el que la sinonimia es una relación discreta, muchas veces se recurre a categorías intermedias (también discretas) como la sinonimia incompleta o la sinonimia parcial (Lyons, 1968).

En cuanto a los segundos (Arppe y Järvikivi, 2007), la idea es que la sinonimia es una relación de semejanza entre dos piezas léxicas que va desde el 0 (ausencia absoluta de semejanza) hasta el 1 (identidad). Desde esta perspectiva, la pregunta sobre si los sinónimos existen no es pertinente y se sustituye por la de cuán sinónimos son dos ítems determinados ${ }^{3}$. Ahora bien, como en todo estudio sobre un objeto de naturaleza gradual, se puede establecer un límite (del tipo de las isoglosas de los dialectólogos) -pongamos el 0,5-rebasado, el cual se puede considerar (entendiendo lo que quiere decir con ello) que un ítem es o no sinónimo de otro.

En esta investigación, uno de los objetivos es tratar de encontrar algún indicio sobre la naturaleza de la sinonimia: si se trata de una relación discreta, de tal modo que las parejas sinónimas y las no-sinónimas se comportan de modo totalmente distinto (en los extremos de un continuo, por ejemplo) o si por el contrario es una relación gradual, de modo que se encuentran parejas en todo el espectro. Lo anterior, para el presente trabajo, interesa tanto desde el punto de vista de la interpretación consciente (metalingüística) como procedimental (de procesamiento automático de la información) del hablante.

\section{2. ¿Es realista la percepción metalingüística de los hablantes?}

Desde un punto de vista metalingüístico, los hablantes de una lengua tienen conciencia de la existencia de la sinonimia. No sólo porque se les puede preguntar directamente por ella (en pruebas de elicitación, del tipo "diga Ud. todos los sinónimos que recuerde de esta palabra" o en pruebas de discriminación, del tipo “‘son sinónimas estas palabras?”), sino también porque es un mecanismo frecuentemente utilizado por los hablantes para evitar la repetición. En este ámbito surge el segundo objetivo de este trabajo, que no es otro que saber si existe (o no) alguna relación entre lo que los hablantes consideran con respecto a la relación de sinonimia y el modo en el que procesan en tiempo real esta relación.

\section{3. ¿Cómo se procesa la relación de sinonimia en el cerebro?}

Un asunto central resulta, además, considerar cómo se procesa la sinonimia en el

\footnotetext{
${ }^{3}$ En este ámbito es crucial el trabajo de Cruse (2000), para el que "synonyms are words 1) whose semantic similarities are more salient tan their differences, 2) that do not primarily contrast with each other; and 3) whose permisible differences must in general be either minor, backgrounded, or both" (Cruse, 2000:160).
} 
cerebro del hablante ${ }^{4}$. Para centrar el problema se partirá de la existencia de un lexicón mental en el que el usuario del lenguaje almacena de algún modo toda la información relevante de las unidades léxicas que no se puede derivar. De este modo, parte de la información sobre cómo se pronuncia una palabra, por ejemplo, pertenece al lexicón mental, dado que no hay otro modo de predecirla. Por el contrario, determinados comportamientos de la pieza léxica (como su posición en la oración, por ejemplo), no se deben incluir en dicho lexicón, pues se derivan de reglas generales de la lengua. Desde este planteamiento, la relación de sinonimia se puede interpretar, bien como una determinada información lexicalizada, bien como derivada de un proceso de comparación en tiempo real.

En el caso de que se trate de una información lexicalizada, varias son las propuestas sobre cómo se produce dicha lexicalización. (i) Una posibilidad, tal vez la más ingenua, es que se trate de una etiqueta que se adquiere con la misma unidad léxica ("del tipo: [sinónimo de x]"); (ii) otra posibilidad, sin embargo, es que los sinónimos se almacenen formando redes, de tal modo que la forma de "lexicalizar" esta información sería la construcción misma de dicha red.

Por otra parte, en el caso de que se trate de una información resultado de un proceso en tiempo real, dicho proceso también puede entenderse de diversas formas. Así, por ejemplo, (iii) podría ser un proceso de comparación de rasgos, de tal modo que los hablantes, al ser expuestos a dos ítems léxicos, compararan rasgo por rasgo hasta decidir si esas dos piezas son o no sinónimas (Katz, 1972); (iv) por el contrario, es posible que el proceso de comparación, lejos de ser atomizado rasgo a rasgo, sea holístico y dependa, por ejemplo, de la posición de cada uno en una determinada red semántica (Quillian, 1968).

Nótese que la posibilidad que se ha denominado (ii) y la (iv) son dos opciones teóricas muy distintas con respecto a la relación de sinonimia. A pesar de que en ambas se acepta la propuesta de Collins y Quillian (1969) de que el léxico se organiza en redes, en la primera de ellas se considera la existencia de redes específicas de sinonimia, de tal modo que lo que se considera cuando se comparan dos ítems en tiempo real es la inclusión o no de ambos en la misma red (es, por ello, que se trata de una información lexicalizada). Por el contrario, la opción que se ha presentado como (iv) interpreta que al decidir si dos unidades son sinónimas éstas se comparan teniendo en cuenta sus relaciones semánticas en el seno de una red compleja. Aquí no hay redes específicas para la sinonimia y la decisión se realiza comparando los ítems entre ellos. De este modo (iv) -al igual que (iii) y a diferencia de las dos primeras hipótesis- asume que una relación léxica como la sinonimia es un tipo de información consciente, en la que el hablante recupera distinta información

${ }^{4}$ El lector interesado en la localización cerebral del procesamiento de la sinonimia puede consultar el trabajo de Jeon, Lee, Kim y Cho (2009), en el que afrontan este asunto con técnicas de neuroimagen. 
lingüística almacenada y la compara entre sí (Murphy, 2003)5.

\section{MÉTODO}

Para dar respuesta a las preguntas que se han ido formulando en el apartado anterior, se preparó un experimento conductual consistente en dos tareas consecutivas: un experimento de decisión semántica y un cuestionario.

\subsection{Participantes}

En este estudio participaron 43 sujetos adultos: 31 mujeres y 12 hombres, estudiantes del Grado de Filología Hispánica de la Facultad de Filosofía y Letras de la Universidad de Zaragoza. Todos ellos participaron de forma voluntaria y recibieron una bonificación de 0,2 puntos sobre su nota final. Todos eran hablantes nativos del español y no tenían problemas de visión sin ajustar.

\subsection{Materiales}

\subsubsection{Tarea de decisión semántica}

Se tomaron las sesenta parejas de palabras sinónimas, de verbos y adjetivos empleadas en el experimento del estudio de Recarte y Peraita (1988). Según se indica en el citado estudio, se trata de veinte parejas de palabras con un alto grado de sinonimia, veinte con un grado medio de sinonimia y otras veinte con un grado bajo de sinonimia. En cada pareja, la primera de las palabras se tomará en lo sucesivo como palabra guía de la sinonimia.

En segundo lugar, se buscaron sesenta palabras distractoras para formar otros sesenta pares de palabras que no eran sinónimas. Para ello, de cada palabra guía se encontraron tres palabras de la misma categoría, con el mismo número de letras y con una frecuencia de uso lo más similar posible. Esta búsqueda y la elección de palabras distractoras se realizó a través de la página Web $N I M^{\natural}$, que indica para

${ }^{5}$ Dentro de la información metalingüística, no obstante, es posible hacer una escala de procesos en virtud de la cantidad de reflexión consciente necesaria. En este sentido, según López García, Montaner, Morant y Pruñonosa (2011), la sinonimia parece menos reflexiva que otras relaciones léxicas como la hiponimia.

${ }^{6}$ Los datos se obtuvieron de la página Web NIM: the stimuli search engine done by and for psycholinguists. Disponible en http://psico.fcep.urv.es/utilitats/nim/eng/valoresesp.php. Consultado el 02/03/2015. 
cada palabra su frecuencia de uso. En la Tabla I se muestra el ejemplo de la palabra guía "denegar" con sus tres palabras distractoras y los datos de número de letras, frecuencia, categoría, etc. Además, se buscaron diez parejas de palabras sinónimas y diez de palabras no sinónimas diferentes de las que se iban a utilizar en el experimento, para el desarrollo del pre-test que sirvió de ensayo a los sujetos.

Tabla I. Ejemplo de palabras distractoras de denegar.

\begin{tabular}{|l|l|l|l|l|l|}
\hline Word & Relative_F & Log & Absolute_F & Letters & POS \\
\hline denegar & 0,711 & 0,233 & 4 & 7 & vrb \\
\hline validar & 0,36 & 0,13 & 2 & 7 & vrb \\
\hline plasmar & 0,89 & 0,28 & 5 & 7 & vrb \\
\hline caldear & 0,53 & 0,19 & 3 & 7 & vrb \\
\hline
\end{tabular}

Una vez que se hubo preparado el material de las parejas de palabras, se creó la plantilla para el programa DMDX, que es el que se utilizó para la realización del experimento y para medir los tiempos de reacción de los participantes. Primero se introdujeron las veinte parejas de palabras de prueba y, a continuación, las ciento veinte parejas de palabras del experimento. Para ello, se hicieron seis grupos de veinte parejas, en cada uno de los cuales se introdujeron diez parejas sinónimas (incluyendo los tres grados de sinonimia) y diez de palabras no-sinónimas. En la plantilla del programa DMDX se especificó que en cada grupo las veinte parejas de palabras se aleatorizaran. Todas estas medidas se realizaron para asegurar que todos los tipos de parejas de palabras aparecieran en todos los tramos del experimento, con el objetivo de evitar sesgos por el cansancio de los participantes. Esta parte del estudio se realizó con un ordenador portátil de tipo netbook de la marca Acer, con un teclado externo.

\subsubsection{Cuestionario}

Para la segunda parte del estudio se utilizó un cuestionario realizado con una aplicación de Google Drive. Se preparó con las mismas ciento veinte parejas de palabras (sesenta sinónimas y sesenta no-sinónimas) del experimento descrito anteriormente. Se introdujeron las ciento veinte parejas de palabras en el cuestionario en un orden aleatorio mezclando parejas de palabras sinónimas y no-sinónimas, mostrando debajo de cada pareja de palabras una escala del 0 al 10. Esta prueba se realizó con un ordenador de sobremesa y un ratón externo para seleccionar el valor para cada ítem. 


\subsection{Procedimiento}

El experimento lo llevaron a cabo los investigadores y tuvo lugar los días 20, 25 y 26 de marzo de 2015 en una habitación aislada del sótano de la Facultad de Filosofía. Cada participante realizó de forma individual las dos pruebas en la misma sesión. Primero realizó el experimento de tiempos de reacción (o prueba de verificación de la sinonimia) y seguidamente contestó el cuestionario (o prueba de juicio de la sinonimia). En total, cada sesión duró alrededor de 30 minutos.

A cada participante se le explicó, antes de empezar la sesión, lo que iba a encontrar y lo que debía hacer. En primer lugar se le advirtió que iba a ver aparecer sucesivamente en la pantalla del ordenador portátil una serie de parejas de palabras y que tenía que decidir si eran o no sinónimas. Para indicar que sí, tenía que pulsar la tecla situada a la derecha de la barra espaciadora del teclado externo y para indicar que no tenía que pulsar la tecla situada a la izquierda de la barra espaciadora. Tenía que pulsar la barra espaciadora para pasar al siguiente ítem a valorar. Se le indicó que se iban a medir los tiempos de reacción, por lo que tenía que contestar lo más rápido posible. También se le informó de que, antes de comenzar la tarea, iba a realizar veinte ensayos de entrenamiento en las mismas condiciones del experimento. Finalmente, se le indicó que no debía ser demasiado estricto a la hora de aceptar la sinonimia (para evitar que sistemáticamente dijera que no) y que era mejor que contestase según su primera impresión.

Los participantes debían completar el cuestionario en el que estaban las parejas de palabras del experimento conductual y para las cuales tenían que indicar el grado de sinonimia que consideraban que tenían dichas parejas, siendo 0 ningún grado de sinonimia y 10 el máximo grado de sinonimia. Para la realización de esta parte, los sujetos sabían que disponían del tiempo que necesitasen para contestar el cuestionario (no se iban a medir los tiempos de reacción esta vez). Una vez contestados todos los ítems, el participante tenía que pulsar en la pantalla el botón "Enviar" para que se guardasen sus respuestas. En ese momento, la sesión experimental habría finalizado.

Así, cada participante escuchó la explicación de la primera prueba que tenía que realizar, realizó la prueba de verificación de la sinonimia empezando por los veinte ítems de ensayo y continuando con las ciento veinte parejas de palabras sinónimas - de los tres grados- y no-sinónimas. Una vez terminada esta parte, se le explicó en qué consistía la segunda prueba, y tuvo que decidir para cada uno de los ciento veinte pares el grado de sinonimia. En este caso, sin límite de tiempo.

\section{RESULTADOS}

Una vez finalizado el periodo de recogida de datos, se obtuvieron dos documentos con resultados: a partir del experimento realizado con el programa DMDX, se 
recibió un archivo con todos los tiempos de reacción de los distintos sujetos; por otra parte, a partir del cuestionario elaborado con la herramienta de Google Drive se obtuvo una planilla Excel con todas las puntuaciones que los distintos sujetos otorgaban a los diferentes ítems.

Con esta información se realizaron los siguientes análisis: en primer lugar, sobre los cuestionarios (3.1.) se analizaron las medias obtenidas por cada uno de los tipos de sinonimia / no-sinonimia y se compararon entre sí. En segundo lugar, en la prueba de juicios sobre la sinonimia (3.2.), se analizó el porcentaje de respuestas discrepantes con el diseño de los materiales (3.2.1.). Además, se compararon los tiempos de reacción de los distintos tipos de sinonimia y se analizó la correlación existente entre las medias de estos tiempos y los tipos de sinonimia (3.2.2.).

Para la realización de estos análisis se utilizaron todos los datos obtenidos por las dos fuentes mencionadas, a excepción de los datos correspondientes a una pareja de no-sinónimos (ganar-creer) que se tuvo que descartar ya que había un error en la plantilla del programa DMDX empleado para la realización del experimento, por lo que no se obtuvieron para ese ítem resultados que se pudieran emplear en el análisis. Por este motivo, se decidió no trabajar con los datos de las mismas parejas de palabras obtenidas del cuestionario. Además, se tuvo que retirar una de las parejas de sinónimos (elegida al azar: veraz, leal) para seguir teniendo el mismo número de ítems en ambas condiciones.

\subsection{Cuestionarios}

Con los resultados obtenidos de los cuestionarios se calculó, para cada grupo, la media de las puntuaciones otorgadas por los participantes. Una vez obtenidas, se compararon estas medias otorgadas a los supuestos sinónimos con las de los supuestos no-sinónimos. Tal y como se muestra en el Gráfico 1, los sinónimos han recibido una puntuación media superior al cinco y los no-sinónimos una muy inferior.

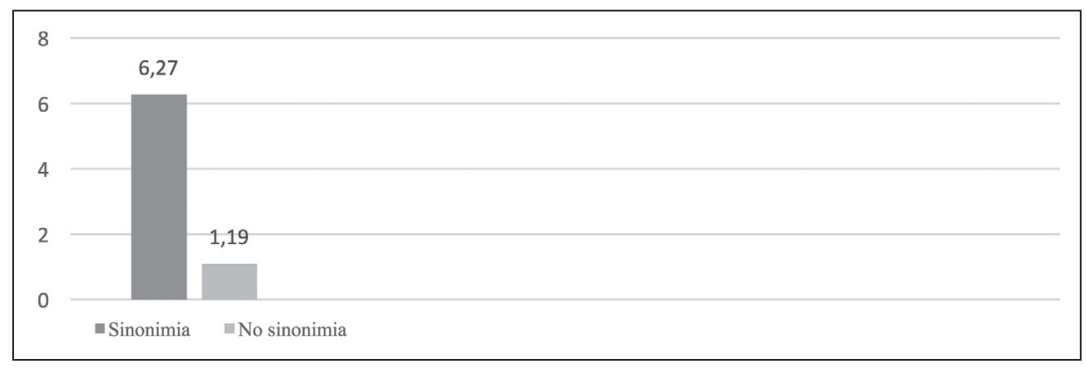

Gráfico 1. Medias de la puntuación obtenida por los grupos de parejas de palabras sinónimas y de las no-sinónimas. 
Para comprobar que la diferencia entre estas medias era significativa, se utilizó el estadístico Prueba de Rangos de Signos de Wilcoxon para muestras pareadas, dado que las muestras no son normales (tal y como indica el test de ShapiroWilk). Como resultado, se obtuvo un p valor de 1.16e-08 (menor que el valor de significancia 0,05 ), por lo que se puede rechazar la hipótesis nula y afirmar que las diferencias entre estas medias sí son significativas.

En segundo lugar, se comprobó si también existía esta diferencia de medias entre los tres niveles de sinonimia planteados por Recarte y Peraita (1988) para los materiales de su estudio. Los resultados se muestran en el Gráfico 2. De nuevo se vio que se cumplían las diferencias entre los grupos de palabras utilizadas. Además, al realizar el análisis de las medias obtenidas para las parejas de palabras no-sinónimas, se apreció una clara diferencia entre las puntuaciones que los sujetos habían atribuido a unas parejas de palabras y a otras, de modo que se pudieron diferenciar dos grupos de no-sinónimos de 30 ítems cada uno, que en el Gráfico 2 se han diferenciado como no-sinónimos altos, aquellos con una puntuación comprendida entre el 0 y el 0,8, y no-sinónimos bajos, con una puntuación superior a $0,8^{7}$.

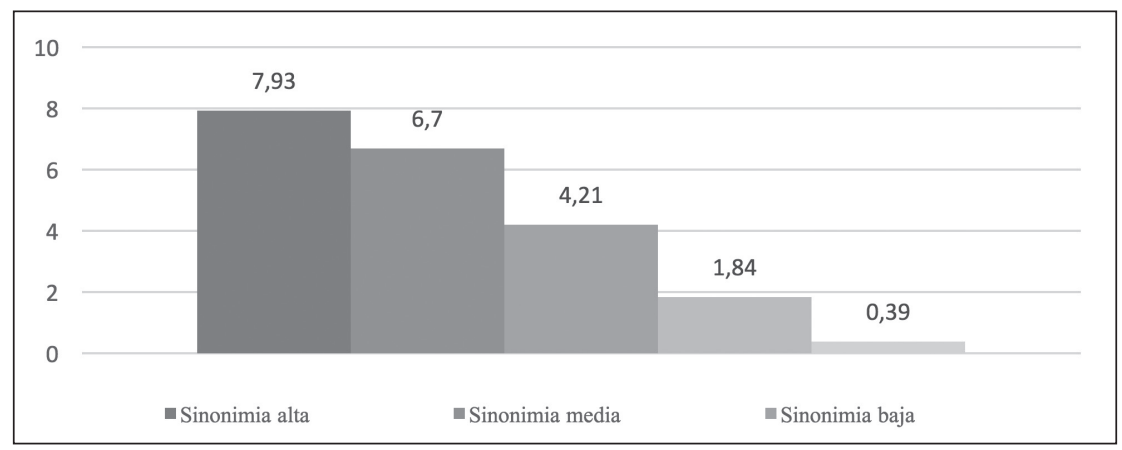

Gráfico 2. Medias de la puntuación obtenida por cada grupo de parejas.

De nuevo en esta ocasión se utilizó el estadístico Prueba de Rangos de Signos de Wilcoxon para muestras pareadas entre los distintos tipos de sinónimos. En la Tabla II se presentan los resultados del p-valor de cada caso:

\footnotetext{
${ }^{7}$ Si se comparan con los resultados obtenidos por Recarte y Peraita 25 años antes, hay que decir que en términos generales es similar (se establecen los mismos grupos entre los sinónimos), si bien las puntuaciones son por lo general más altas en el artículo de los años ochenta $(8,9 ; 7,67$ y 6,09 respectivamente). En aquel estudio no se realizaron dos grupos entre los no-sinónimos, pues se consideraron como elementos meramente distractores.
} 
Tabla II. Resultados de la Prueba de Rangos de signos de Wilcoxon para muestras pareadas.

\begin{tabular}{|l|l|l|l|l|l|}
\hline & $\begin{array}{l}\text { Sinónimos } \\
\text { Altos }\end{array}$ & $\begin{array}{l}\text { Sinónimos } \\
\text { Medios }\end{array}$ & $\begin{array}{l}\text { Sinónimos } \\
\text { Bajos }\end{array}$ & $\begin{array}{l}\text { No Sinónimos } \\
\text { Altos }\end{array}$ & $\begin{array}{l}\text { No Sinónimos } \\
\text { Bajos }\end{array}$ \\
\hline Sinónimos Altos & - & $4.189 \mathrm{e}-08$ & $<2.2 \mathrm{e}-16$ & $1.16 \mathrm{e}-08$ & $1.16 \mathrm{e}-08$ \\
\hline Sinónimos Medios & $4.189 \mathrm{e}-08$ & - & $1.159 \mathrm{e}-08$ & $1.157 \mathrm{e}-08$ & $2.274 \mathrm{e}-13$ \\
\hline Sinónimos Bajos & $<2.2 \mathrm{e}-16$ & $1.159 \mathrm{e}-08$ & - & $1.157 \mathrm{e}-08$ & $1.16 \mathrm{e}-08$ \\
\hline No Sinónimos Altos & $1.16 \mathrm{e}-08$ & $1.157 \mathrm{e}-08$ & $1.157 \mathrm{e}-08$ & - & $1.705 \mathrm{e}-08$ \\
\hline No Sinónimos Bajos & $1.16 \mathrm{e}-08$ & $2.274 \mathrm{e}-13$ & $1.16 \mathrm{e}-08$ & $1.705 \mathrm{e}-08$ & - \\
\hline
\end{tabular}

Como se refleja en la Tabla II, todos los p-valores son menores que el valor de significancia $(0,05)$, por lo que en todos los casos se puede rechazar la hipótesis nula y afirmar que las diferencias entre estas medias sí son significativas.

\subsection{Tarea de decisión semántica y comparación de ambas pruebas}

\subsubsection{Porcentaje de juicios discrepantes}

El primer aspecto que se analizó sobre los resultados de esta tarea conductual fue el porcentaje de juicios emitidos por los participantes que resultaron ser diferentes con respecto al planteamiento que se tuvo a la hora de diseñar los materiales. Es decir, se quiso ver el porcentaje de respuestas en el que los participantes contestaron "No" a una pareja que fue considerada pareja de sinónimos durante el diseño del experimento, y viceversa, el porcentaje de respuestas que consideraron sinónimas parejas de palabras distractoras. En el Gráfico 3 se muestran los resultados obtenidos y en el Gráfico 4 los porcentajes de errores:

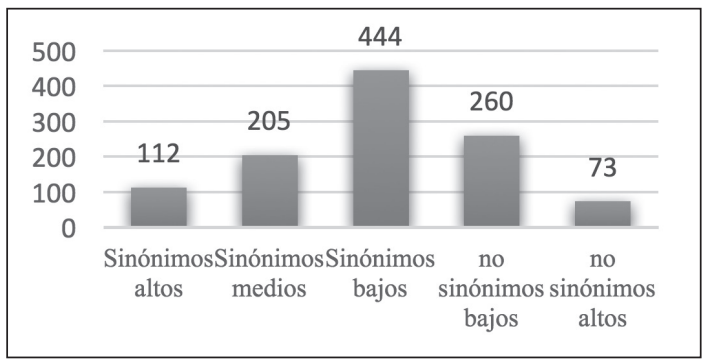

Gráfico 3. Discrepancias entre los juicios emitidos por los participantes y el diseño de los materiales. 


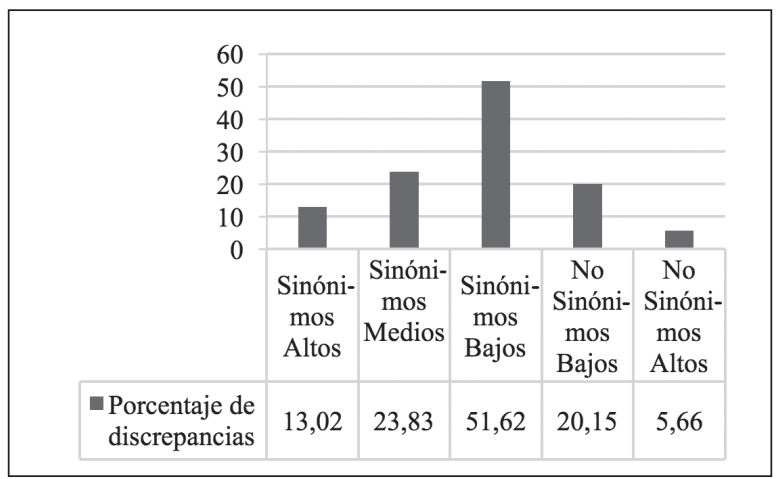

Gráfico 4. Porcentaje de discrepancias entre los juicios emitidos por los participantes y el diseño de los materiales.

Como se observa en los Gráficos 3 y 4, a mayor nivel de sinonimia, menor porcentaje de discrepancia con los materiales. Del mismo modo, en una relación especular, cuanto menos sinónimos son dos ítems, menos respuestas discrepantes hay.

Para saber si las diferencias entre los porcentajes obtenidos son significativas se realizó un Test Exacto de Fisher. En la Tabla III se muestran los resultados. Como se ve, son todos significativos, salvo la diferencia entre no sinónimos bajos y sinónimos medios.

Tabla III. Resultados del Test Exacto de Fisher. Valores de p.

\begin{tabular}{|l|l|l|l|l|l|}
\hline & $\begin{array}{l}\text { Sinónimos } \\
\text { Altos }\end{array}$ & $\begin{array}{l}\text { Sinónimos } \\
\text { Medios }\end{array}$ & $\begin{array}{l}\text { Sinónimos } \\
\text { Bajos }\end{array}$ & $\begin{array}{l}\text { No Sinónimos } \\
\text { Bajos }\end{array}$ & $\begin{array}{l}\text { No Sinónimos } \\
\text { Altos }\end{array}$ \\
\hline Sinónimos Altos & ---- & $8.693 \mathrm{e}-09$ & $2.2 \mathrm{e}-16$ & $1.596 \mathrm{e}-05$ & $4.188 \mathrm{e}-09$ \\
\hline Sinónimos Medios & $8.693 \mathrm{e}-09$ & ----- & $2.2 \mathrm{e}-16$ & 0.04783 & $2.2 \mathrm{e}-16$ \\
\hline Sinónimos Bajos & $2.2 \mathrm{e}-16$ & $2.2 \mathrm{e}-16$ & ---- & $2.2 \mathrm{e}-16$ & $2.2 \mathrm{e}-16$ \\
\hline No Sinónimos Bajos & $1.596 \mathrm{e}-05$ & 0.04783 & $2.2 \mathrm{e}-16$ & ---- & $2.2 \mathrm{e}-16$ \\
\hline No Sinónimos Altos & $4.188 \mathrm{e}-09$ & $2.2 \mathrm{e}-16$ & $2.2 \mathrm{e}-16$ & $2.2 \mathrm{e}-16$ & ----- \\
\hline
\end{tabular}

\subsubsection{Tiempos de reacción}

En segundo lugar se analizaron los resultados del experimento conductual. En concreto, se compararon las medias del tiempo de reacción (TR) en los diferentes grupos de parejas de palabras. En el Gráfico 5 se presentan los resultados de las palabras sinónimas frente a las no sinónimas. 


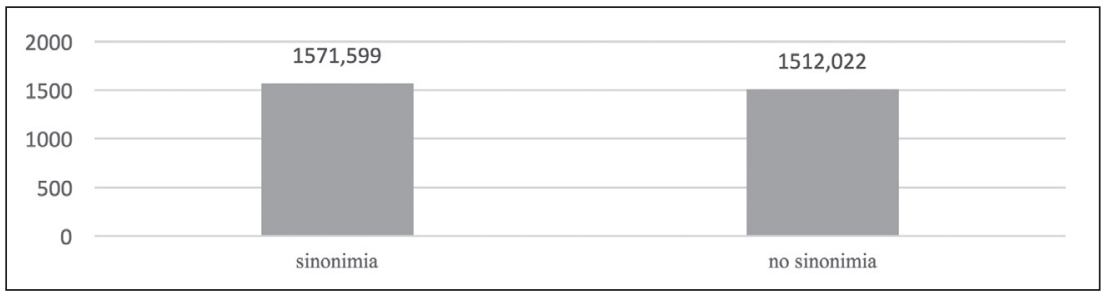

Gráfico 5. Medias de los tiempos de reacción de las parejas de palabras sinónimas frente a las no-sinónimas.

Para comprobar que la diferencia entre estas dos medias era estadísticamente significativa, se empleó el estadístico de la T de Student para muestras pareadas. Se ha utilizado este estadístico una vez que se ha comprobado que las muestras tienen distribución normal (mediante el test de Shapiro-Wilk). Los resultados muestran un p-valor de 0.003148 , lo que indica que se puede afirmar que las medias son distintas con significación estadística.

A continuación, consideramos las diferencias entre los distintos grupos de sinónimos y no sinónimos. Los resultados aparecen en el Gráfico 6:

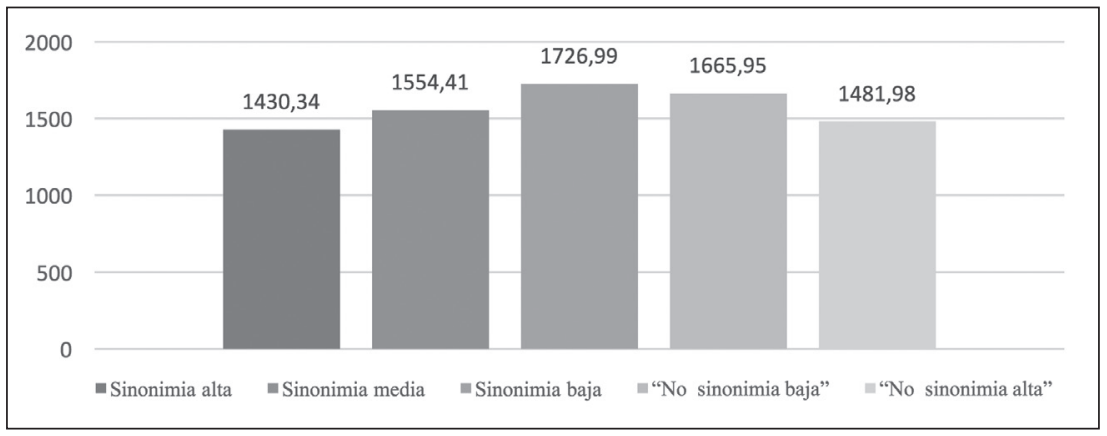

Gráfico 6. Medias de los tiempos de reacción en las distintas categorías de (no)-sinónimos.

Para medir la significación de las medias de los distintos tipos de Sinónimos y No Sinónimos se utilizó el estadístico de T-Student para muestras pareadas. En la Tabla IV se reflejan los resultados obtenidos: 
Tabla IV. Resultados del Test Exacto de Fisher. Valores de p.

\begin{tabular}{|l|l|l|l|l|l|}
\hline & $\begin{array}{l}\text { Sinónimos } \\
\text { Altos }\end{array}$ & $\begin{array}{l}\text { Sinónimos } \\
\text { Medios }\end{array}$ & $\begin{array}{l}\text { Sinónimos } \\
\text { Bajos }\end{array}$ & $\begin{array}{l}\text { No Sinónimos } \\
\text { Bajos }\end{array}$ & $\begin{array}{l}\text { No Sinónimos } \\
\text { Altos }\end{array}$ \\
\hline Sinónimos Altos & - & $1.743 \mathrm{e}-06$ & $2.547 \mathrm{e}-13$ & $2.22 \mathrm{e}-05$ & 0.979 \\
\hline Sinónimos Medios & $1.743 \mathrm{e}-06$ & - & $9.269 \mathrm{e}-12$ & 0.2519 & $4.209 \mathrm{e}-05$ \\
\hline Sinónimos Bajos & $2.547 \mathrm{e}-13$ & $9.269 \mathrm{e}-12$ & - & $9.013 \mathrm{e}-06$ & $1.201 \mathrm{e}-13$ \\
\hline No Sinónimos Bajos & $2.22 \mathrm{e}-05$ & 0.2519 & $9.013 \mathrm{e}-06$ & - & $3.185 \mathrm{e}-11$ \\
\hline No Sinónimos Altos & 0.979 & $4.209 \mathrm{e}-05$ & $1.201 \mathrm{e}-13$ & $3.185 \mathrm{e}-11$ & - \\
\hline
\end{tabular}

Como se puede ver en la Tabla IV, todos los resultados son significativos salvo en la comparación de los no sinónimos altos y los sinónimos altos por un lado y los sinónimos medios y los no sinónimos bajos por otro.

Por último, se calculó la correlación entre el tiempo de reacción de los participantes durante la prueba de verificación de la sinonimia (con independencia de si la respuesta había sido la esperada o no) y el grado de sinonimia que habían atribuido a cada pareja de palabras en la prueba de juicio de la sinonimia. Para la obtención de estos resultados se calculó una correlación lineal de Pearson y una regresión lineal. Para ello, se utilizó como base la lista de resultados de tiempos de reacción que entregó el mismo programa DMDX con el que se realizó la prueba de verificación de la sinonimia. El programa proporcionó la información ordenándola por sujeto e indicando en cada caso la fecha y hora de realización del experimento y el tiempo de reacción empleado por el sujeto experimental para cada ítem.

En el caso del coeficiente de correlación de Pearson, se ha obtenido un p-valor más pequeño que 2,2 e- 16 . Así, como es más pequeño que 0,05 , se puede afirmar que existe correlación entre ambas variables.

Para medir la bondad del ajuste realizado con la regresión lineal se ha obtenido el coeficiente de determinación, con un valor igual a 0,07758 . Como es un valor cercano a 1 , significa que existe una relación lineal suficiente. En cuanto al contraste de regresión, se obtiene un p-valor más pequeño que 2,2 e-1. Esto vuelve a indicar que se rechaza la hipótesis nula (la no influencia lineal de la variable independiente).

\section{DISCUSIÓN Y CONCLUSIONES}

Después de haber obtenido los resultados del análisis, se puede atender a las preguntas que motivaron el presente estudio. 


\subsection{La naturaleza gradual o discreta de la sinonimia}

Para contestar a esta pregunta se consideró, en primer lugar, las medias obtenidas en el cuestionario. En el caso de que la relación de sinonimia fuera discreta (dos palabras son o no son sinónimas), se esperaría que las medias otorgadas a las parejas de palabras se situaran en los extremos de la escala, obteniendo una puntuación cercana al 10 todas aquellas parejas sinónimas y una puntuación cercana al 0 las no-sinónimas. Por el contrario, en el caso de que la relación fuera gradual, se observaría una distribución a lo largo de la escala, de tal modo que se tendrían parejas tanto en los extremos como en el centro de la misma. Si se consideran los resultados obtenidos del cuestionario (v. Gráfico 2) se ve claramente cómo reciben una puntuación más alta los sinónimos considerados altos $(7,93 / 10)$ y cómo esta puntuación va bajando en la sinonimia media $(6,11 / 10)$ y en la sinonimia baja, donde obtiene una puntuación de 4,41/10. Además, se pudo diferenciar entre dos grupos de no-sinónimos de 30 ítems cada uno cuyas puntuaciones son significativamente distintas. Esta diferencia entre los dos grupos de palabras no-sinónimas refuerza la idea de la gradualidad de la sinonimia. Según estos resultados, se puede decir que los hablantes no distinguen un límite nítido entre la sinonimia y la nosinonimia, sino que perciben esta relación semántica como un continuo.

Si se observan ahora los resultados obtenidos durante la realización del experimento, específicamente el porcentaje de discrepancias, si la sinonimia fuera una relación discreta, cabría esperar que la unanimidad o la discrepancia estuvieran distribuidas de manera homogénea entre todas las parejas de palabras empleadas en el estudio. Si por el contrario, la sinonimia fuera una relación gradual, cabría esperar que hubiera más unanimidad en las parejas de palabras situadas en los extremos (sinonimia alta, media y no-sinonimia alta), y que hubiera más discrepancias en la zona central en la que se situarían las parejas de la sinonimia baja y de la no-sinonimia baja.

En los resultados obtenidos (v. Gráficos 3 y 4) se observa que el porcentaje de discrepancias en la respuesta de los sujetos a la hora de decidir si una pareja era o no sinónima también es gradual: va aumentado desde los sinónimos altos $(13,02 \%$ de discrepancias en las respuestas), pasando por los sinónimos medios $(23,83 \%)$ hasta los sinónimos bajos $(51,62 \%$ de discrepancias $)$ para volver a disminuir en los no-sinónimos bajos $(20,15 \%)$ y alcanzar el mínimo porcentaje de discrepancia en los no-sinónimos altos (5,66\% de discrepancias). Se encuentra así una zona intermedia que abarcaría desde los sinónimos medios hasta los nosinónimos bajos donde los sujetos habrían diferido más en sus respuestas, y frente a esto, se tendrían dos extremos en los que los participantes habrían tenido un porcentaje de mayor unanimidad a la hora de decidir si dos palabras eran sinónimas o no. Esta diferencia en la heterogeneidad-homogeneidad de las respuestas apoya, como es posible ver, la hipótesis de la naturaleza gradual de la sinonimia. De hecho, es interesante señalar que todos los grupos son estadísticamente dife- 
rentes salvo los sinónimos medios y los no sinónimos bajos, que deben tener un nivel similar de incertidumbre.

También en los resultados del experimento es importante tener en cuenta los tiempos de reacción. Si la relación de sinonimia fuera discreta, sería esperable encontrar tiempos de reacción homogéneos en todas las parejas de palabras. En cambio, si fuera una relación gradual, cabría encontrar tiempos de reacción heterogéneos según los tipos de parejas de palabras.

En relación a esto, se observa de nuevo un comportamiento gradual, de modo que el tiempo de reacción va aumentado desde la sinonimia alta (1430,34 ms) hasta la sinonimia baja $(1726,99 \mathrm{~ms})$, se mantiene elevada en la no-sinonimia baja $(1665,95 \mathrm{~ms})$ y desciende otra vez en la no-sinonimia alta $(1481,98 \mathrm{~ms})-\mathrm{v}$. Gráfico 6-. Se encuentra, por tanto, un patrón de comportamiento similar al del porcentaje de discrepancia en el que existiría una zona central en la que los hablantes tardarían más en tomar la decisión a la hora de juzgar si una pareja de palabras era o no sinónima, y en los dos extremos (que se corresponderían con la sinonimia alta y con la no-sinonimia alta) los hablantes tardarían menos tiempo, lo que se puede traducir en que tendrían menos dudas a la hora de decidir. En este caso, se han encontrado diferencias significativas entre todos los grupos, salvo en dos (v. Tabla 4): los sinónimos altos y los no sinónimos altos, por un lado, y los sinónimos medios y los no sinónimos bajos, por otro. Esta falta de diferencias significativas incide en el carácter especular que se ha encontrado.

Como conclusión, se puede considerar que, a la luz de los resultados de esta investigación, todo parece indicar que la relación de sinonimia es gradual y no discreta. Esto es, que no tiene sentido preguntarse si dos entradas léxicas son o no sinónimas, sino que la pregunta pertinente debería ser, en todo caso, cuán sinónimas son dos entradas léxicas.

Ante esto, queda por explicar por qué los tiempos de reacción de los participantes son estadísticamente distintos si se trata de sinónimos o de no sinónimos (siendo más lentos los tiempos de decisión en parejas sinónimas) -v. Gráfico 5-. La explicación de este resultado se cree que está en el diseño de materiales, dado que sólo se tienen 20 parejas de sinonimia alta, frente a los 30 pares de no-sinónimos altos. Además, en las 20 parejas de sinonimia baja, la imprecisión es mayor, frente a los 30 no sinónimos bajos, cuyos valores se relacionan en mayor medida con la sinonimia media.

En definitiva, se cree que el presente estudio avala que la relación de sinonimia es una relación gradual.

\section{2. ¿La consciencia metalingüística de los hablantes es realista?}

En relación a si la consciencia de los hablantes sobre la sinonimia correlaciona con 
el procesamiento automático de la misma, se calcularon una correlación de Pearson y una regresión lineal entre las respuestas obtenidas en el cuestionario (en la escala Likert de 0 a 10) y los tiempos de reacción obtenidos en la tarea conductual. Si la consciencia metalingüística de los hablantes correlacionaba con el procesamiento automático de la sinonimia, se obtendría un valor de "p" menor o igual a 0,05 y si no correlacionaban, el valor de "p" sería mayor a 0,05. Como indican los resultados, se aprecia que la relación entre el tiempo de reacción y el grado de sinonimia es una relación estadísticamente significativa. Según estos resultados, se puede considerar que la consciencia metalingüística de los hablantes -registrada por las puntuaciones obtenidas en el cuestionario- y el procesamiento automático de la sinonimia -registrado por los tiempos de reacción del experimento-, sí que están relacionados entre sí. En concreto, como se comentó, los resultados indican que cuanto más clara está la relación desde el punto de vista consciente (metalingüístico: valor asignado en el cuestionario), menos tiempo cuesta tomar la decisión (experimento).

\subsection{Semántica léxica, memoria y sinonimia}

Por último, se puede considerar qué consecuencias tienen los resultados obtenidos sobre la naturaleza de la sinonimia. Por un lado, hay que admitir que la sinonimia es una relación significativa que opera tanto a nivel de reflexión consciente como en el tiempo de reacción, decidiendo más deprisa las relaciones de sinonimia alta que las dudosas. Por otra parte, el hecho de haber comprobado que la relación de sinonimia es gradual especular y no discreta, lleva a plantearse cuestiones de almacenamiento y de procesamiento de la información. Que sea gradual implica que no se trata de una etiqueta según la cual dos palabras son sinónimas o no lo son, sino que implica un proceso de comparación en tiempo real. Es una decisión semántica que los hablantes realizan. En lo referido a la comparación, cabe preguntarse qué es lo que comparan los hablantes para considerar si dos palabras son o no sinónimas. Obviamente, en este estudio no es posible responder esta pregunta en profundidad, pero no se ha querido perder la oportunidad de considerar qué indican los resultados obtenidos al respecto.

Si el proceso de comparación consistiera en un proceso serial, en el que se fueran comparando uno a uno los rasgos semánticos de cada una de las piezas léxicas, se habrían encontrado que los denominados "sinónimos altos" tendrían los tiempos de reacción más altos de todos (pues la decisión se pospondría al final de la lista de rasgos, mientras que el resto habrían encontrado discrepancias antes). Sin embargo, los resultados obtenidos van en la dirección contraria y muestran una relación inversa especular: los hablantes son más rápidos cuanto más alta es la sinonimia o cuanto más alta es la no-sinonimia, y son más lentos en tomar la 
decisión en las zonas intermedias. Esto implica -a nuestro juicio- que la comparación que se está realizando no es serial, sino holística. Se comparan los dos ítems de forma global.

Un modelo que podría encajar con esta propuesta de comparación holística sería el modelo cognitivo que enfoca el estudio del léxico desde una perspectiva psicológica. En este modelo, la especificidad de las unidades léxicas no sería discreta, sino que estaría sujeta a un continuum que iría de lo más específico a lo más esquemático. De hecho, un elemento léxico representa una categoría compleja ya que no tiene un único significado sino una variedad de sentidos con varios grados de afianzamiento. Estos diferentes sentidos de un elemento léxico forman una red y se relacionan con otras redes mediante los procedimientos de extensión o de elaboración (Mateu Fontanals, 2009: 282-287). Aplicando estos conceptos al presente trabajo, se puede entender que la gradualidad a la hora de identificar a dos unidades léxicas como sinónimas puede estar basada en este concepto de redes semánticas. En concreto, se podría considerar, con Zapico y Vivas (2014), que la sinonimia es un tipo de relación basada en la distancia semántica. Esto explicaría que el tiempo de reacción fuese menor en la sinonimia alta y en la no-sinonimia alta, ya que serían unidades léxicas con redes que estarían o bien muy cerca o muy lejos de la red de la palabra guía en la memoria semántica. En cambio, costaría más tiempo tomar la decisión para los casos de sinonimia media ya que las redes estarían a una distancia intermedia de la red de la palabra guía. Del mismo modo se explicaría que los sinónimos y no-sinónimos altos tengan menor nivel de discrepancia que los sinónimos y no-sinónimos bajos y medios. Tanto en los casos de mucha cercanía semántica como en los casos de mucha distancia, la decisión es más unánime y rápida que en los casos intermedios.

\section{CONCLUSIONES}

En este trabajo se ha tratado de responder a algunas de las preguntas más relevantes que plantea el estudio de la sinonimia desde una perspectiva psicolingüística. Para ello, se ha seguido un procedimiento experimental ya planteado 25 años atrás en el trabajo de Recarte y Peraita (1988), si bien el análisis de los resultados en este estudio ha sido parcialmente distinto.

Como conclusión de este estudio, se puede afirmar que nuestra investigación sugiere que la sinonimia es una relación continua y gradual, de tal modo que todos los ítems léxicos están relacionados entre sí en una escala de 1 (identidad) a 0 (ausencia de semejanza). Esta relación, que opera de forma automática (es más rápido y más eficiente comparar sinónimos y no-sinónimos altos que bajos), también es observable a nivel de reflexión consciente, pues el porcentaje de sinonimia que otorgan los sujetos en una escala Likert correlaciona con las medidas encontradas 
en el experimento de decisión semántica. Por último, este estudio también sugiere que el contenido semántico de los ítems léxicos se desprende a través de su red de relaciones y no de unos supuestos rasgos o primitivos internos. Esta propuesta está en consonancia, además, con trabajos como los de De Almeida (1999), según los cuales los ítems más complejos no parecen ser más pesados que los ítems más simples.

Quedan, sin embargo, algunas preguntas sin contestar. Al entender la sinonimia como un proceso metalingüístico (esto es, de reflexión consciente) sobre la distancia semántica, se debería considerar cómo distinguirla de otros tipos de relaciones como la hiponimia o la antonimia, por ejemplo. De igual manera, se debería investigar si es cierto, como afirman Hino, Lupker y Pexman (2002), que las palabras con muchos sinónimos se procesan más despacio y, de ser cierto, por qué ocurre. En el modelo al que parecen apuntar los datos obtenidos en la presente investigación, la sinonimia es un proceso de comparación en tiempo real, por lo que la cantidad de sinónimos que una entrada léxica posea en un sistema concreto no debería influir en su procesamiento. Pero ésos son temas ya de una próxima investigación.

\section{REFERENCIAS}

Arppe, Antti y Järvikivi, Juhani. (2007). Every method counts: combining corpusbased and experimental evidence in the study of synonymy. Corpus Linguistics and Linguistic Theory, 3(2), 131-159.

Casas Gómez, Miguel. (1995). Sinonimia y eufemismo. Quaderni di Semantica, $16,17-46$.

Collins, Allan y Quillian, M. Ross. (1969). Retrieval time from semantic memory. Journal of Memory and Language, 8(2), 240.

Cruse, Alan. (2000). Meaning in language. An introduction to Semantics and Pragmatics. Oxford: Oxford University Press.

De Almeida, Roberto. (1999). The representation of lexical concepts: A psycholinguistic inquiry. Unpublished PhD Dissertation. New Brunswick, NJ: Rutgers University.

Fruyt, Michèle. (1994). Typologie des cas de synonymie en latin, C. Moussi (ed.), Les problèmes de la synonimie en latin, Paris: PUPS, 25-46.

García-Hernández, Benjamín (1997). Sinonimia y diferencia de significado. Revista española de lingüística, 27(1), 1-31.

Gauger, Hans-Martin. (1972). Zum Problem der Synonyme, Tübinga: G. Narr.

Glynn, Dylan y Justina Robinson. (2014). Corpus Methods for Semantics: Quantitative studies in polysemy and synonymy, 43, 7, 205-238.

Gries, Stefan T. y Otani, Naoki. (2010). Behavioral profiles: A corpus-based per- 
spective on synonymy and antonymy. ICAME Journal, 34, 121-150.

Hino, Yasushi; Lupker, Stephen J. y Pexman, Penny M. (2002). Ambiguity and Synonymy Effects in Lexical Decision, Naming, and Semantic Categorization Task: Interactions Between Ortography, Phonology, and Semantics, Journal of Experimental Psychology: Learning, Memory and Cognition, 28.4, 686-713. Jeon, Hyeon-A; Lee, Kyoung-Min; Kim, Young-Bo y Cho, Zang-Hee. (2009). Neural substrates of semantic relationships: common and distinct left-frontal activities for generation of synonyms vs. antonyms, Neuroimage, 48(2), 449457.

Katz, Jerrold J. (1972). Semantic Theory. New York: Harper and Row.

López García, Ángel; Montaner, Amparo; Morant, Ricardo y Pruñonosa, Manuel. (2011). Redes léxicas como redes neuronales, Revista de Investigación Lingüistica 14, 61-86.

Lyons, Johns. (1968). Introduction to Theoretical Linguistics, Cambridge: Cambridge University Press.

Mateu Fontanals, Jaume. (2009). Modelos Cognitivos. Miguel, Elena de (ed). Panorama de la lexicología, Barcelona: Ariel, 281-300.

Milner, Jean-Claude. (1989). Introduction a une science du langage, Paris, Seuil.

Murphy, M. Lynne. (2003). Semantic relations and the lexicon: antonymy, synonymy and other paradigms, Cambridge: University Press.

Quillian, M. Ross. (1968). Semantic memory, in M. Minsky (ed.), Semantic Information Processing, 227-270.

Recarte Goldaracena, Miguel Ángel y Herminia Peraita Adrados. (1988). Tipos de verificación de la sinonimia: implicaciones para las teorías de la memoria semántica. Cognitiva, 1(3), 229-243.

Salvador, Gregorio. (1985). "Sí hay sinónimos", en Semántica y lexicología del español. Estudios y lecciones, Madrid, Paraninfo, 51-66.

Trujillo, Ramón. (1996). Principios de Semántica Textual, Madrid, Arco/Libros.

Zapico Martín y Jorge Vivas. (2014). La sinonimia como caso particular de distancia semántica, Encontros Bibli: Revista Eletrônica de Biblioteconomia e Ciência da Informação, 19(40): 253-266. 